5.

DERECHO ADMINISTRATIVO 



\title{
LAS ASOCIACIONES PÚBLICO-PRIVADAS EN LA UNIÓN EUROPEA: ELEMENTOS PARA UN ANÁLISIS SOBRE LA CONCESIÓN DE OBRA PÚBLICA EN CHILE
}

[Public-Private Partnerships in the European Union: Facts to Analyze the Concession of Public Works in Chile]

\author{
Martín Loo GutiérREZ* \\ Universidad Adolfo Ibáñez, Viña del Mar, Chile
}

\begin{abstract}
RESUMEN
El trabajo expone las razones utilizadas por los órganos políticos de la Unión Europea para incentivar el uso, por parte de sus Estados miembros, de instrumentos de colaboración entre el sector público y el sector privado en el desarrollo infraestructural y en la prestación de servicios públicos. Con ello presenta perspectivas que ayuden a la construcción de un discurso justificativo en Chile de esta modalidad de intervención del sector privado en ámbitos de relevancia pública.

\section{Palabras clave}

Asociaciones público-privadas Concesiones de obras públicas.
\end{abstract}

\begin{abstract}
This work sets forth the reasons put forward by the European Union's political bodies to foster the use, by its member States, of collaboration tools between the public and the private sectors in terms of infrastructure development and in the rendering of public services. In doing so, it provides approaches to assist in the construction of a justifying speech in Chile of this mode of intervention of the private sector in areas of public relevance.

KeYwords

Public Private Partnerships -Concessions of Public Works.
\end{abstract}

RECiBIDO el 22 de abril y ACEPTADO el 7 de diciembre de 2015

* Profesor asistente en la Facultad de Derecho de la Universidad Adolfo Ibáñez. Doctor en derecho público por la Universidad de Roma - Tor Vergata. Licenciado en ciencias jurídicas por la Pontificia Universidad Católica de Valparaíso. Correspondencia: Avenida Padre Hurtado 750, Viña del Mar, Chile. Email: martin.loo@ uai.cl 


\section{INTRODUCCIÓN}

Durante su discurso ante el IV Congreso Nacional de la Asociación Gremial de Concesionarios de Obras de Infraestructura Pública, celebrado en Santiago en octubre de 2014, la presidente de la República hizo explícito el interés de su gobierno en "consolidar la legitimación de la cooperación público-privada, no sólo en quienes creemos [en ella], sino también ante la ciudadanía". Más allá del significado que la presidente haya querido atribuir a la palabra "legitimación", elaborar un discurso justificativo (o "legitimador") de este método de realización de infraestructuras y prestación de servicios públicos demanda necesariamente una mejor comprensión de las razones que se encuentran detrás de él.

Con este artículo se intentará aportar algunos argumentos que sirvan para la construcción de dicho discurso justificativo. Esto se hará en el bien entendido que la política nacional de concesiones de obras públicas es, con naturales matices, transversal a las principales orientaciones ideológicas presentes en nuestro Congreso Nacional. Para enfrentar esta tarea se tomará como punto de referencia la reflexión que se desarrolló en Europa a partir de la publicación del "Libro Verde sobre la colaboración público-privada, la contratación pública y las concesiones”, del año 2004, proceso concluido recientemente con la publicación de la primera directiva comunitaria sobre los contratos de concesión ${ }^{2}$.

Esta investigación, por tanto, sólo pretende exponer sistemáticamente las razones que la Unión Europea tomó en consideración para impulsar a sus Estados miembros a utilizar una categoría de relaciones jurídicas entre el sector público y el sector privado conocida como "public private partnerships", o sea, nuestras "asociaciones público-privadas", con el objeto de aprovechar las capacidades financieras y organizacionales del sector privado en tareas tradicionalmente asumidas por el sector público, como son el desarrollo infraestructural y la prestación de servicios públicos. No pretende, por ende, someter a examen crítico la plausibilidad de las razones esgrimidas por la Unión Europea ni aquellas otras que podrían ser utilizadas en la elaboración del discurso justificativo de nuestro propio sistema concesional.

Antes de comenzar es necesario aclarar una cuestión terminológica de alguna relevancia. En el lenguaje de la Unión Europea, la expresión “asociación público-privada" es un término genérico que comprende diversas

1 "Nota de prensa de la Presidencia de la República de Chile del 23 de octubre de 2014", disponible en www.prensapresidencia.cl

${ }^{2}$ Directiva 2014/23/CE, de 26 de febrero de 2014, relativa a la adjudicación de contratos de concesión (Diario Oficial de la Unión Europea de 28 de marzo de 2014). 
figuras, unas contractuales y otras propiamente organizativas, entre las cuales se cuenta la concesión de obras públicas. En razón de ello, la distinción más importante que hacen los instrumentos de la Comisión Europea sobre el tema, es aquella que divide estas asociaciones público-privadas en asociaciones "contractuales" (como las concesiones de obras, los proyectos de financiamiento privado, etc.) e "institucionales" (como las sociedades de economía mixta). Por el contrario, en nuestro país, el término "concesión de obras públicas" comprende una serie de figuras que, en Europa, serían consideradas asociaciones público-privadas de "tipo contractual", pero calificadas como algo diverso de la concesión propiamente tal. Para evitar confusiones, aún a riesgo de parecer impreciso, se utilizarán indistintamente los términos "concesión” y "asociación público-privada".

\section{ANTECEDENTES}

Tal como sucedió en nuestro país, durante los años noventa del siglo XX, la Unión Europea dio inicio a una política de incentivo de la participación del sector privado en la realización de infraestructuras de uso o utilidad pública y en la prestación de "servicios de interés general"3.

Con esta política ${ }^{4}$, la Unión Europea buscaba que sus Estados miembros limitaran la importancia del sector público en tales ámbitos abriendo más espacios a la participación del sector privado en dichas áreas de la economía. Todo lo anterior se demostraba necesario ya que servicio público y sector público, en casi todos los países miembros de la Comunidad, incluso a fines del siglo XX, seguían "confundidos", como consecuencia del importante

${ }^{3}$ La expresión "servicios de interés general”, por genérica que pueda parecer, no es casual. La doctrina sostiene que ella reemplazó en el "idioma" del derecho comunitario, la expresión "servicio público". Sin embargo, su significado y las razones detrás del 'abandono' de la expresión "servicio público" no pueden ser objeto de este trabajo. Sobre el punto, véase SORDI, Bernardo, Servizi pubblici e concorrenza: su alcune fibrillazioni tra diritto comunitario e tradizione continentale, en Quaderni Fiorentini per la Storia del Pensiero Giuridico Moderno, 31 (2002), p. 578. En el presente artículo se utilizarán indistintamente las expresiones "servicio público" y "servicio de interés general", en el entendido que, cuando se utilice la primera, se usa la expresión en su sentido funcional y no orgánico, que son los dos sentidos que, según nuestra doctrina, reconoce el término.

${ }^{4} \mathrm{La}$ doctrina sostiene que el interés que manifiesta la Unión Europea por este tipo de relaciones contractuales entre la administración pública y los particulares es de naturaleza política; cosa que debe tenerse en consideración al momento de 'explicar' estos mecanismos de provisión de infraestructuras y servicios. Véase CHITI, Mario Pilade, I partenariati pubblico-privati e la fine del dualismo tra diritto pubblico e diritto comune, en El Mismo (editor), Il partenariato pubblico-privato. Concessioni, finanza di progetto, società miste, fondazioni (Napoli, Editoriale Scientifica, 2009), p. 2. 
rol que el Estado había adquirido en la gestión de los servicios prestados a la población 5 .

La inspiración de esta política se encuentra en la convicción de los órganos comunitarios sobre el hecho que, si la Administración del Estado recurría a la experiencia negocial del sector privado, mejoraría la eficiencia de la construcción y la calidad de la gestión y explotación de las infraestructuras de servicio público, especialmente (aunque no únicamente), desde el punto de vista del gasto fiscal.

La política comunitaria en cuestión encontraba sus antecedentes en la necesidad de realizar ciertos proyectos de infraestructura que, según los órganos comunitarios, eran indispensables para el crecimiento de la economía continental. La ejecución de tales proyectos, según la Unión, no podría llevarse a la realidad sin la colaboración del sector privado ${ }^{6}$.

El interés político por contar con una reglamentación de nivel continental que facilitara la realización de proyectos de infraestructura pública comenzó a tomar forma con la adopción de una serie de actos comunitarios tanto de tipo normativo (directivas), como no normativo (libros verdes) ${ }^{7}$.

Algunas de las directivas han tenido como objeto de regulación, pre-

${ }^{5}$ Mameli, Barbara, Servizio pubblico e concessione. L'influenza del mercato unico sui regimi protezionisti e regolamentati (Milano, Giuffrè, 1998), p. xix.

${ }^{6} \mathrm{Se}$ trata de los proyectos conocidos como "Redes Transeuropeas de Transporte". En el documento de la Comisión de las Comunidades Europeas titulado "Desarrollo de la red transeuropea de transporte: Financiaciones innovadoras, interoperabilidad del telepeaje”, COM (2003) 132 Final, de 23 de abril de 2003, quedó de manifiesto el alto nivel de consenso político que tenía la realización de estas infraestructuras y el decidido favor con el cual la Comisión Europea miraba la participación privada en su financiación, realización y explotación.

${ }^{7}$ Las directivas son, a todos los efectos, normas positivas de derecho supranacional que los Estados miembros deben incorporar en sus ordenamientos jurídicos (artículos 289-291 del Tratado sobre el Funcionamiento de la Unión Europea). Dicha incorporación es obligatoria en cuanto a los fines y "libre" en cuanto a los medios a través de los cuales se realice (artículo 288, inciso $3^{\circ}$ TFUE). En caso que el respectivo Estado incumpla su obligación de incorporar las normas de la directiva ellas se "auto-incorporan" en virtud de su «efecto directo». Los libros verdes ("green papers") son, por su lado, actos de la Comisión Europea, carentes de fuerza normativa, en los cuales se expone y analiza el estado del arte sobre la materia de que traten y se plantean preguntas (en la práctica, un cuestionario) que sirven para estimular la reflexión de las partes interesadas, normalmente, los operadores de un mercado, las autoridades reguladoras, las asociaciones de consumidores, etc. Su publicación inaugura un proceso de consultas públicas durante el cual los sujetos involucrados por la futura y eventual regulación son invitados a hacer valer su punto de vista sobre las propuestas contenidas en el documento. Tal proceso culmina con la publicación de un documento de análisis de las respuestas recibidas, el cual puede servir de base para la formulación de una propuesta legislativa. 
cisamente, los procedimientos que los Estados miembros deben observar para la adjudicación de los contratos públicos. El fin principal de estas reglas es la armonización de las legislaciones internas incluso en materias que pueden aparecer tan "locales" como la contratación administrativa ${ }^{8}$.

La función meramente consultiva que distingue a los libros verdes no debe inducir a menospreciar su importancia dentro del procedimiento legislativo comunitario ya que, aun careciendo de fuerza normativa, los resultados de las consultas públicas a que dan pie pueden llegar a tener una considerable influencia en los procesos de reforma legislativa.

Esto fue lo que ocurrió en el asunto que nos atañe. En abril de 2004, sólo un mes después de la adopción de dos importantes directivas referidas a la coordinación de los procedimientos de adjudicación de ciertos contratos del Estado", la Comisión Europea publicó un "Libro Verde sobre la colaboración público-privada y el derecho comunitario en materia de contratación pública y concesiones" ${ }^{\prime 10}$. Con él se inició el proceso de debate sobre la necesidad de introducir una regulación común europea sobre concesiones y otras formas de colaboración entre el Estado y el sector privado, materias que habían sido excluidas de las directivas aprobadas en marzo de dicho año.

Luego de un largo proceso de discusión y análisis, en el mes de enero de

${ }^{8}$ Podría pensarse que la actividad contractual de las administraciones públicas es un área del ordenamiento jurídico en la cual no debiera caber el ejercicio de la potestad normativa supranacional que compete a la Unión. Se podría afirmar que, siendo la contratación del Estado una materia de exclusivo interés nacional, tiene un limitado impacto en el sistema económico comunitario. Tales conclusiones serían, sin embargo, erradas. En efecto, el derecho comunitario tiene una fuerte incidencia en la normativa nacional sobre contratación pública ya que tal actividad de la Administración es clave (y no solamente por su relevancia económica) para la efectiva vigencia de los principios fundamentales del mercado interno, como son los principios relacionados a las "cuatro libertades fundamentales" (libertad de circulación de mercancías, servicios, trabajadores y capitales: art. 26.2 TFUE). En este sentido, véase, Frilet, Marc - Lager, Florent, Public Procurement Issues in the European Union, en European Business Law Review, 21 (2010), p. 399; Bovis, Christopher, EU Public Procurement Law (Cheltenham - Northampton, Edward Elgar Publishing, 2007), p. 4; García Capdepón, Pedro María, Los contratos de colaboración público-privada, en Revista española de la función consultiva, 3 (2005), p. 105; y DEL SAZ, Silvia, La nueva ley de contratos del sector público. ¿Un nuevo traje con las mismas rayas? en Revista de Administración Pública, 174 (2007), p. 343.

${ }^{9}$ Directivas números 2004/17/CE y 2004/18/CE, del 31 de marzo de 2004.

${ }^{10}$ Comisión de las Comunidades Europeas, Libro verde sobre la colaboración público privada y el derecho comunitario en materia de contratación pública y concesiones COM (2004) 327 Final, del 27 de abril de 2004, de ahora en adelante, citado como "Libro Verde sobre la CPP". 
2011, la Comisión Europea presentó el "Libro Verde sobre la modernización de la política de contratación pública de la Unión Europea. Hacia un mercado europeo de la contratación pública más eficiente”, COM (2011) 15 def. En él se planteó derechamente la necesidad de introducir reformas a las directivas sobre contratación pública del 2004 con el objeto, entre otros, de facilitar los procedimientos de adjudicación de los contratos públicos y permitir que ellos puedan convertirse en mecanismos para coadyuvar a la satisfacción de otras políticas comunitarias (inclusión social, respeto medioambiental, etc.). Posteriormente, en diciembre de 2011, la Comisión Europea llevó ante el Parlamento Europeo dos propuestas legislativas: la primera, titulada "Propuesta de Directiva del Parlamento Europeo y del Consejo relativa a la Contratación Pública”, COM (2011) 896 Final, proponía la adopción de nuevas normas sobre contratación pública y la derogación de las directivas 17 y 18 del 2004; la segunda, titulada "Propuesta de Directiva del Parlamento Europeo y del Consejo relativa a la adjudicación de contratos de concesión”, COM (2011) 897 Final, planteó la necesidad de adoptar una norma comunitaria sobre concesiones.

Finalmente, en febrero de 2014, el proceso iniciado en abril de 2004 con la publicación del Libro Verde sobre la CPP, culminó con la aprobación de las directivas 2014/23, 2014/24 y 2014/25. La primera, como ya se señaló, establece normas sobre la adjudicación de los contratos de concesión. Las dos últimas establecen normas sobre la contratación pública y derogan las directivas 2004/18 y 2004/17. Con la aprobación de estas directivas se concluyó el prolongado proceso de revisión de la disciplina comunitaria sobre contratación pública. La Unión Europea cuenta hoy con una reglamentación completa sobre la contratación del Estado que se extiende, también, a las concesiones de obras públicas.

Como puede advertirse, el origen de la reflexión comunitaria sobre este fenómeno de colaboración entre la Administración Pública y el sector privado, se encuentra en el ya citado Libro Verde sobre la CPP. En este documento, la Comisión expone las razones que, según su concepto, justifican que se intensifique el uso de la colaboración privada en el desarrollo infraestructural y en la prestación de servicios públicos.

Las ideas contenidas en dicho instrumento, traducidas en la nueva directiva del 2014, pueden utilizarse para ilustrar las razones que podrían servir de justificación a nuestro propio sistema de concesiones de obras públicas. La sistematización de estas razones es necesaria toda vez que nuestra doctrina jurídica asume como auto-evidente la "bondad" de la participación del sector privado en el desarrollo de proyectos de infraes- 
tructura pública, cuestión que contrasta con la amplia investigación que, economistas e ingenieros, han dedicado al argumento ${ }^{11}$.

En el apartado siguiente analizaremos cuáles son dichas ideas.

\section{ORÍGENES DE LA POLÍTICA COMUNITARIA DE INCENTIVO}

DE LA COLABORACIÓN ENTRE SECTOR PÚBLICO Y PRIVADO

\section{Endeudamiento público excesivo y mayor disciplina fiscal.}

Una de las motivaciones de esta política es la limitada capacidad presupuestaria de los Estados para hacer frente a las necesidades de inversión en proyectos de infraestructura que, por su naturaleza, requieren una importante inversión inicial que las administraciones públicas - sometidas a una rigidez presupuestaria siempre mayor - no pueden satisfacer recurriendo al "viejo" mecanismo del endeudamiento público" ${ }^{12}$.

La estrechez presupuestaria del sector público europeo encuentra una explicación, al menos parcial, en el principio establecido por el art. 126, inc. $1^{\circ}$, del Tratado sobre el funcionamiento de la Unión Europea, conforme al cual "los Estados miembros evitarán déficits públicos excesivos". Esta norma se tradujo, en 1997, en el Pacto de Estabilidad y Crecimiento ${ }^{13}$ que obligó a los Estados europeos a revisar sus políticas de endeudamiento público debiendo, entre otras medidas, modificar su política de inversiones en infraestructuras. Esto llevó a que se disminuyera el recurso a la deuda pública para el financiamiento de proyectos infraestructurales y a la búsqueda de asociaciones con empresas privadas dispuestas a asumir su ejecución, financiamiento y explotación ${ }^{14}$.

Pero, ¿cómo obtener que los Estados de la Unión Europea continuaran construyendo las infraestructuras necesarias para el desarrollo económico $\mathrm{y}$, al mismo tiempo, contuvieran el endeudamiento fiscal?

${ }^{11}$ Un solo ejemplo, muy reciente, sirva como justificación: ENGEL, Eduardo y otros, The Economics of Public-Private Partnerships. A Basic Guide (New York, Cambridge University Press, 2014).

${ }^{12}$ Bovis, Christopher, The Development of Public Private Partnerships at European Level, en European Public Private Partnership Law Review (2006), p. 1.

${ }^{13}$ Consejo Europeo, Resolución del Consejo sobre el Pacto de estabilidady crecimiento (Amsterdam, 17 de junio de 1997), Diario Oficial de las Comunidades Europeas del 2 de agosto de 1997. Con dicho pacto, los países de la unión monetaria acordaron que sus Estados miembros no podrían acumular una deuda pública superior al $60 \%$ del producto interno bruto ni presentar un déficit presupuestario superior al $3 \%$ del mismo producto interno.

${ }^{14}$ Dorrego de Carlos, Alberto - Martínez Vásquez, Francisco, La colaboración público-privada en Los MISMOS (editores), La colaboración público-privada en la Ley de contratos del sector público (Madrid, La Ley, 2009), p. 72. 
La respuesta llegó, en febrero de 2004, desde la Oficina de Estadísticas Europea (Eurostat), bajo la forma de una decisión que modificó el sistema de contabilidad unificado europeo ("European System of National and Regional Accounts", "ESA 95”), justamente, en la parte relativa a la contabilización de las inversiones del Estado realizadas con motivo de ciertos contratos públicos denominados "public-private partnerships", esto es, nuestras "asociaciones público-privadas".

La decisión señala que Eurostat había detectado que los Estados miembros, con creciente frecuencia, recurrían a dichas modalidades de contratación pública con el objetivo de evitar registrar en el balance fiscal algunas de sus inversiones en infraestructura (manteniéndolas, se decía, "fuera de los registros"). Por medio de este mecanismo contable, los Estados europeos buscaban limitar el impacto que tenían tales inversiones en las cuentas públicas. Eurostat, notando que la falta de reglas uniformes contribuía a que estas inversiones públicas recibieran un tratamiento contable heterogéneo, decidió introducir modificaciones en el sistema de contabilidad tendientes a establecer una regulación más precisa y uniforme.

Según la decisión, el tratamiento contable que debía darse a la inversión realizada por el Estado dependería del modo en el cual las partes del acuerdo (genéricamente, sector público y sector privado) repartieran los riesgos que surgen del proyecto. Con tal finalidad, la decisión distingue tres tipos de riesgos: riesgo de construcción (en la fase de ejecución de la infraestructura), riesgo de disponibilidad y riesgo de demanda (propios de la fase de explotación de la misma).

Sobre la base de este distingo, la decisión autorizó a los Estados miembros a no registrar en el balance público las inversiones que realizaran cuando, en conformidad con los acuerdos contractuales, fuera posible concluir que el riesgo de construcción y el riesgo de demanda o el riesgo de disponibilidad se encontraban asignados a la gestión y control del contratista privado. Sólo en este caso, el sector público se encontraría autorizado a mantener la inversión que debía realizar fuera de la contabilidad pública. De este modo se limitaba la repercusión de tal inversión en el nivel de endeudamiento y de eventual déficit público, contribuyendo con ello al respeto del principio establecido en el ya citado art. 126 , inciso $1^{\circ}$, TFUE ${ }^{15}$.

Como se puede intuir, los mecanismos de asociaciones público-privada

${ }^{15}$ Eurostat, Long Term Contracts Between Government Units and Non-Government Partners (Public-Private Partnerships), 2004 y Eurostat, Press realease 18/2004: New Decision of Eurostat on Deficit and Debt: Treatment of Public-Private Partnerships, 11-02-2004, especialmente, p. 2: "the assets involved in a public-private partnership can be considered as non-government assets only if there is strong evidence that the partner is bearing most of the risk attached to the specific partnership". 
ganaron "popularidad" en la Unión Europea debido a que el tratamiento contable que les fue reservado permitía eludir las severas normas que prohíben a los países de la unión monetaria incurrir en endeudamiento y/o en déficit público excesivo.

Es a ello que se debe, entonces, el inmediato interés que algunos Estados demostraron por este tipo de contratos públicos luego de la publicación del Libro Verde sobre la CPP, del $2004^{16}$. Algunos Estados europeos, de hecho, justificaron su decisión de incorporar reglas sobre estas modalidades contractuales en sus legislaciones, precisamente, por los beneficios que representaba para las cuentas fiscales la posibilidad de ejecutar infraestructuras sin tener que registrar la inversión en el balance público ${ }^{17}$.

Sin embargo, una aproximación de ese tipo deja de tomar en cuenta los demás aspectos de esta modalidad de provisión de infraestructura pública. En efecto, como intentaremos analizar en el siguiente apartado, el interés político por la participación privada encuentra justificación, también, en la mejora de la eficiencia de los servicios públicos y de la calidad del gasto fiscal.

\section{Mejores servicios públicos, mejor gasto fiscal.}

Una segunda razón que explica la atención que esta clase de acuerdos suscitó en la Unión Europea, se encuentra en la posibilidad que se abre para el sector público de aprovechar la experiencia negocial acumulada por el sector privado en la ejecución y explotación de proyectos de infraestructuras y servicios. Al respecto, la Comisión Europea sostuvo en el Libro Verde sobre la CPP que las modalidades de trabajo del sector privado pueden revelarse útiles para la consecución de las finalidades de interés público de la Administración del Estado ${ }^{18}$.

${ }^{16}$ Nótese que la decisión Eurostat es de febrero de 2004, las nuevas directivas sobre contratación pública, de marzo de 2004 y el libro verde sobre la colaboración público-privada, de abril de 2004. Estos instrumentos constituyen un bien coordinado esquema de intervención política en favor de las asociaciones público-privadas.

${ }^{17} \mathrm{La}$ aproximación que identifica sólo una utilidad contable en la figura contractual, ha sido criticada por la doctrina de aquellos países donde se ha manifestado en forma más evidente, como es el caso de Italia. Véase, Cori, Rosalba, Giorgiantonio, Cristina - PARADISI, Ilaria, Allocazione dei rischi e incentivi per il contraente privato: un' analisi delle convenzioni di project financing in Italia, en AA.VV., Le infrastrutture in Italia: dotazione, programmazione, realizzazione (Roma, Banca d'Italia, 2011), pp. 259-288, especialmente, p. 260.

18 "Libro Verde sobre la CPP”, párr. 3: "Diferentes factores explican el recurso cada vez más frecuente a las operaciones de CPP. [...] [una de ellas] radicaría en el deseo de aprovechar en mayor medida los conocimientos y métodos de funcionamiento del sector privado en el marco de la vida pública”. 
Parte de las ideas que la Comisión Europea expuso en el Libro Verde sobre la CPP se inspiran en la experiencia del Reino Unido donde - a partir del primer gobierno de Margaret Thatcher - ganó fuerza la política conocida como "New Public Management", en cuyo marco surgió el programa de desarrollo denominado "Private Finance Initiative". Este sistema de contratación es el directo antecedente de la colaboración público-privada objeto del Libro Verde sobre la CPP ${ }^{19}$.

En el marco ideológico que inspiró el New Public Management, el contrato (público) pasó a convertirse en un instrumento de concreción del interés colectivo, ya que el sector privado se transformó en un relevante suministrador de servicios públicos. Al respecto, la doctrina sostiene que "la ideología del NPM [...] significó un cambio de modelo de la gestión pública próxima al modelo de gestión privada" ${ }^{20}$, dando lugar al fenómeno de la "administración a través de contratos"21.

Como ya se dijo, en el ámbito ideológico que sirvió de sustento al New Public Management ${ }^{22}$, se desarrolló el programa denominado Private Finance Initiative, cuyo objetivo central era obtener del sector privado el financiamiento necesario para la ejecución de obras públicas. El Private Finance Initiative es, por ende, uno de los mecanismos activamente utili-

${ }^{19}$ Sobre el "New Public Management", Nieto Garrido, Eva, El "New Public Management" y el Gobierno a través de contratos en el Reino Unido, en Revista de Administración Pública, 162 (2003), pp. 391-417. Sobre la "Private Finance Initiative", NiEto Garrido, Eva, La financiación privada de obras y servicios públicos en el Reino Unido (de Private Finance Initiative a Public Private Partnerships), en Revista de Administración Pública, 164 (2004), pp. 389-406.

${ }^{20}$ Nieto Garrido, El "New Public Management" y el Gobierno a través de contratos en el Reino Unido, cit. (n. 19), p. 394.

${ }^{21}$ Grimshaw, Adam y otros, Going Privately: Partnership and Outsourcing in UK Public Services, en Public Administration, 80 (2002), p. 475: "The emergence of neo-liberal ideology and its critique of the supposed monopolistic and bureaucratic inefficiencies within the public sector culminated in the political project of the New-Right Conservative administration (1979-97). There was widespread privatization of provision of public services, guided by the public-choice model of services consumption. These reforms are well documented and are identified as a shift towards 'quasi-markets', 'post-bureaucratic forms' or 'government by contract'”. (se han omitido las citaciones internas). Sobre la evolución del fenómeno en tiempos a nosotros más recientes, con particular visión crítica de la poca accountability que se presenta en Estados Unidos, véase, FreEman, Jody - Minow, Martha (editores), Government by Contract: Outsourcing and American Democracy (Cambridge, Mass. London, Harvard University Press, 2009).

${ }^{22}$ Yescombe, Edward, Public-Private Partnerships: Principles of Policy and Finance (Oxford, Butterworth Heinemann, 2007), p. 16; Nieto Garrido, El "New Public Management" y el Gobierno a través de contratos en el Reino Unido, cit. (n. 19), p. 392. 
zados por los gobiernos ingleses para incentivar la participación del sector privado en actividades de producción y distribución de servicios públicos ${ }^{23}$.

Pese a que el New Public Management surgió al alero de la ideología conservadora, el programa de Private Finance Initiative alcanzó su pleno desarrollo sólo con los gobiernos laboristas que sucedieron al gobierno de John Major. Fue, entonces, durante los gobiernos de signo político opuesto al de sus ideadores que, como sostiene la doctrina, el Private Finance Initiative pasó a ser "la fórmula preferente para la realización de obras públicas de gran envergadura" en Gran Bretaña ${ }^{24}$. Este hecho demuestra la transversalidad ideológica alcanzada por la política pública en cuestión en el Reino Unido.

Uno de los corolarios más relevantes de la adopción de este sistema de provisión de infraestructura pública se encuentra en la incorporación explícita de instrumentos y criterios de evaluación de la "eficiencia" de los proyectos. En efecto, para entender las razones que tuvo la Unión Europea para promover el uso de este tipo de técnicas contractuales, se debe subrayar la importancia que adquirieron dos expresiones "técnicas" en la justificación política del programa de PFI: value for money y public sector comparator.

La expresión "mejor value for money" es el concepto que traduce la finalidad económica que se pretende alcanzar con el Private Finance Initiative. Su significado podría sintetizarse señalando que consiste en la obtención de los mejores resultados posibles de una determinada inversión. Sin embargo, no se trata únicamente de la obtención de un resultado al menor costo, sino que del mejor resultado al costo más ventajoso posible, lo que no corresponde, necesariamente, al menor desembolso económico.

Para establecer si el "value for money" de un determinado proyecto es "mejor" que el de otro proyecto, similar pero ejecutado con métodos tradicionales de contratación pública, es necesario un modelo de comparación, esto es, de un proyecto hipotético en el cual el sector privado carezca del rol global que le corresponde en un proyecto financiado por

${ }^{23}$ AlLEn, Grahame, The private finance initiative, House of Commons Library Research Paper 03/79 (House of Commons, London, 2003), p. 9-14.

${ }^{24}$ Nieto GarRIDO, La financiación privada de obras y servicios públicos en el Reino Unido (de Private Finance Initiative a Public Private Partnerships), cit. (n. 19), p. 390; Giorgiantonio, Cristina - Giovanniello, Valentina, Infrastrutture e "project financing" in Italia: il ruolo (possibile) della regolamentazione en AA.VV., Le infrastrutture in Italia: dotazione, programmazione, realizzazione (Roma, Banca d'Italia, 2011), p. 227: "Il Regno Unito, oltre a costituire l'ordinamento europeo in cui il PPP contrattuale (nella formula della Private Finance Initiative - PFI) ha trovato la sua prima applicazione, rappresenta anche il paese ove esso ha raggiunto la sua massima diffusione, soprattutto per le opere di maggiore complessità". 
él mismo, como las concesiones: dicho proyecto hipotético se denomina public sector comparator ${ }^{25}$.

Para explicar mejor la relación que existe entre ambas nociones, se puede recurrir a las palabras de la doctrina española, según la cual "la idea que subyace bajo el PFI es la de que el value for money tendrá que ser demostrado mediante la evaluación y el contraste detallados de las ofertas del sector privado con una referencia comparativa del sector público, que suele ser denominada public sector comparator (PSC), a través de la cual se pretende determinar lo que le costaría a la Administración la obtención de los mismos resultados recurriendo a un contratista privado a través de un mecanismo distinto del PFI [...] El PSC es, por tanto, un punto de referencia para comparar las ofertas presentadas en régimen de $\mathrm{PFI}^{\prime 26}$.

De lo anterior es posible concluir que "value for money" y "public sector comparator" son dos expresiones en estrecha relación: el "public sector comparator" es un instrumento que permite comparar el valor de un proyecto realizado por el sector público utilizando contratos públicos tradicionales, con el valor de otro proyecto similar ejecutado con la participación del sector privado en las tareas de financiación, diseño, ejecución y explotación de la infraestructura, con la asunción de los relativos riesgos. Este instrumento permite determinar cuál forma de ejecución del proyecto otorgará un mejor "valor por precio" o "valor al dinero" que el Estado debe invertir. Con esta metodología se buscar establecer cuál utilización de los recursos públicos resultará más eficiente.

La premisa detrás del programa del Private Finance Initiative, que se traslada al sistema concesional europeo de la mano del Libro Verde sobre la CPP, es que - gracias a la participación del sector privado y la transferencia de los riesgos del proyecto que aquel puede gestionar en modo más eficiente - se alcanzaría un mejor "value for money" ${ }^{27}$ que tendrá positivas repercusiones, tanto en el presupuesto público (liberando recursos que serán utilizados en otras finalidades), como en la calidad de los servicios recibidos por los administrados.

La experiencia del Reino Unido en materia de incentivo de la participación del sector privado en la realización de infraestructuras de servicio público ha sido analizada por distintos organismos internacionales, como el

\footnotetext{
${ }^{25} \mathrm{OECD}$, Transport Infrastructure Investment. Options for Efficiency (Paris, OECD Publishing, 2008), p. 24.

${ }^{26}$ Nieto Garrido, La financiación privada de obras, cit. (n. 19), p. 398.

${ }^{27}$ Public-Private Partnerships: In Pursuit of Risk Sharing and Value for Money (Paris, OECD Publishing, 2008), p. 13: "[...] sufficient transfer of risk to the private partner is necessary to ensure efficiency and value for money. For the transfer of risk to be the most effective, risk must also be transferred to the party best able to carry it".
} 
Fondo Monetario Internacional y la Organización para la cooperación y el desarrollo económico $(\mathrm{OCDE})^{28}$. Estas instituciones y la doctrina destacan que las asociaciones público-privadas son un vehículo para mejorar la calidad de las inversiones públicas gracias a la traslación de responsabilidades y riesgos al sector privado ${ }^{29}$.

La OCDE, yendo más allá del sólo análisis "técnico" de la figura contractual, concentra su atención en las consecuencias de tipo "sistémico" que derivan de la decisión política de recurrir a este tipo de relaciones asociativas o colaborativas entre la Administración Pública y el sector privado, identificando en ellas una evidencia de la re-definición de los roles tradicionales del sector público y privado ${ }^{30}$ : aquél, (auto)limitando su rol como productor y titular de los servicios públicos, comienza a actuar como regulador y fiscalizador de la actuación del sector privado que, por su parte, asume el papel de prestador de servicios a la colectividad.

De lo anterior derivaría una significativa alteración de algunas de las más tradicionales concepciones del derecho administrativo. Éste, en cuanto derecho que regula la especial posición que el ordenamiento jurídico ha conferido a un específico sujeto (la Administración Pública) en sus relaciones con los administrados-ciudadanos, así como los diversos privilegios que derivan de la finalidad de su función, particularmente claros en materia de contratación pública, sufre un importante cambio de perspectiva a causa de la necesidad de "reacomodar" los roles de ambos extremos del complejo social, el "público" y el "privado", pasándose desde una visión de naturaleza autoritativa a una de tipo colaborativa ${ }^{31}$.

${ }^{28}$ International Monetary Fund (Fiscal Affairs Department), Public-Private Partnerships (Washington (DC.), 2004); OECD, Public-private partnerships, cit. (n. 27).

${ }^{29}$ International Monetary Fund (Fiscal Affairs Department), cit. (n. ), p. 7; SadKa, Efraim, Public-Private Partnerships: A Public Economics Perspective, SSRN eLibrary (2006); AкIтовY, Bernardin et al., Public Investment and Public-Private Partnerships en International Monetary Fund Economic Issues 40 (2007); HoppeA, Eva I. y Schмiтz, Patrick W., Public-private partnerships versus traditional procurement: Innovation incentives and information gathering, SSRN eLibrary (2012).

${ }^{30}$ OECD, Public-private partnerships, cit. (n. 27), p. 13: «[a]lthough private firms have been involved in public service delivery for a long time, the introduction of public-private partnerships (PPPs) in the early 1990s established a mode of public service delivery that redefined the roles of the public and private sectors»; «[t]he introduction of PPPs also raised a series of political, economic and technical questions. The first issue is whether there should be public or private provision of services that are traditionally provided by the public sector.»

${ }^{31}$ Fundamental para entender la relevancia que ha tenido la contraposición de las categorías de 'lo público' y 'lo privado' en el desarrollo de la dogmática de derecho público es BobBio, Norberto, La grande dicotomia: pubblico/privato en EL MISMO, Stato, governo, società (2a edición, Einaudi, Torino, 1995), p. 3-22; para una lectu- 
Esta reacomodación de algunas de las tradicionales concepciones acerca del derecho administrativo y de la posición institucional de la Administración sería el más importante resultado derivado de estas nuevas modalidades de contratación pública. El complejo fenómeno de colaboración entre el sector público y el privado, tiene relevantes repercusiones en la lectura global del sistema administrativo, las cuales podrían ser sintetizadas afirmando que la posición paradigmática ocupada por los Estados europeos en los procesos económicos de sus países ha variado desde su condición de productor directo de utilidades sociales a regulador de la actividad privada de provisión de tales servicios.

\section{El cambio en el paradigma del rol del Estado en la economía.}

El rol ocupado por el Estado en la economía europea se caracterizó, durante todo el siglo XX, por su constante expansión para luego, a partir de los años conclusivos de la década de los 90, comenzar un proceso de reducción. Este complejo devenir es fruto de numerosos factores históricos, culturales y jurídicos cuyo análisis excede el objeto de este trabajo.

Debe destacarse, sin embargo, que la realización conjunta entre sector público y privado de proyectos de infraestructura y prestación de servicios públicos es una de las manifestaciones de la importante mutación sufrida por el papel del Estado en las sociedades europeas. Tales mutaciones tienen origen en los procesos de integración política y de unificación y liberalización jurídica y económica vividos en el continente con posterioridad a la Segunda Guerra Mundial. Dichos procesos han hecho que el sujeto público abandone el papel protagónico que le correspondió en el sistema económico de Europa occidental durante toda la segunda mitad del siglo $\mathrm{XX}$, obligándolo a renunciar a su posición "monopólica” en la definición y satisfacción del interés general, a abrir posibilidades de participación del sector privado en la satisfacción de necesidades colectivas y, sobre todo, a reconocer al sector privado la condición de actor fundamental en tales finalidades ${ }^{32}$.

ra desde una perspectiva específicamente administrativa, véase Napolitano, Giulio, Pubblico e privato nel diritto amministrativo (Giuffrè, Milano, 2003).

${ }^{32}$ Fioravanti, Maurizio, Per una storia dello Stato moderno in Europa en Quaderni Fiorentini per la Storia del Pensiero Giuridico Moderno 39 (2010), p. 75, quien, desde una perspectiva histórica, explica que el siglo $\mathrm{XX}$, «[...] con il suo carico di tensioni sociali, con le presenze nuove dei partiti politici e dei sindacati, con l'accrescimento complessivo dei compiti dello Stato nella società, e delle presenze della società nello Stato e nella sua amministrazione, rende sempre meno credibile questa versione monolitica dello Stato e della sua genesi, raffigurata come una corsa fatalmente destinata all'esito della esclusività, della conquista totale del campo da parte dello Stato sovrano.» Véase también, González García, Julio V., Contrato de colaboración público- 
El cambio -iniciado en el período entre la Primera y la Segunda Guerras Mundiales ${ }^{33}$ - aumentó su velocidad e intensidad con la promulgación de las constituciones europeas post-bélicas (Francia, 1946; Italia, 1948; Alemania, 1949). En ellas, los Estados de Europa continental asumieron, en modo directo y explícito, ciertos deberes de mejoramiento de las condiciones de vida de sus ciudadanos, reforzando el "Estado de bienestar" que, de ahí a pocos años, se transformaría en el modo más evidente de intervención estatal en la economía ${ }^{34}$.

El proceso post-bélico de reforzamiento del Estado de bienestar conllevó el incremento del número y del tipo de servicios públicos dando lugar al

privada en Revista de Administración Pública 170 (2006), p. 10, según el cual existen "[...] también razones que afectan al papel del Estado en las sociedades del siglo XXI y la incapacidad que parecen tener los poderes públicos para la dotación de todas las infraestructuras necesarias para la sociedad moderna, sobre todo en tiempos de contención del déficit público y de reducción de la presión fiscal, aspecto este último que, sin lugar a dudas, condiciona el recurrir a las fórmulas más tradicionales, como puede ser el contrato de obra pública. Desde determinados ámbitos se defiende que su rol se limite a actividades de regulación y a la provisión de aquellos servicios que, por no proporcionar beneficios, no pueden ser prestados por los particulares.»

${ }^{33}$ Durante los años que siguieron a la Primera Guerra Mundial, en un contexto geográfico y cultural bien definido, se vivió un intenso e indeleble proceso de reformulación dogmática del derecho público en general y administrativo, en particular, fruto del trabajo de los juristas que entre Weimar (la ciudad de la primera constitución democrática de Alemania) y Viena (la ciudad de la Constitución redactada por Hans Kelsen para la República Austriaca), reformularon la lectura tradicional, exclusiva y excluyentemente 'públicística' y autoritativa, del poder público-administrativo. El período histórico y sus profundas secuelas en la dogmática iuspublicística, es magistralmente ilustrado por SoRDI, Bernardo, Tra Weimar e Vienna. Amministrazione pubblica e teoria giuridica nel primo dopoguerra (Giuffrè, Milano, 1987), al cual reenviamos para mayores noticias.

${ }^{34}$ CASSESE, Sabino, La «vecchia» costituzione economica: $i$ rapporti tra Stato ed economia dall Unità ad oggi en EL MISMO (editor), La nuova costituzione economica (4a edición, Laterza, Roma-Bari, 2007), p. 7. Sobre la relación entre el fenómeno del intervencionismo del Estado y las normas y principios contenidos en las "constituciones económicas» de los Estados europeos y sobre cómo ellas han sido materialmente 'modificadas' por los Tratados comunitarios, véase CASSESE, Sabino, La costituzione economica europea en Rivista italiana di diritto pubblico comunitario (2001), pp. 907922 y, en general, los estudios contenidos en el ya citado CASSESE, Sabino (editor), La nuova costituzione economica (4a edición, Laterza, Roma-Bari, 2007). Sobre el surgimiento del fenómeno del «welfare state», al menos en el Reino Unido, véase Wade, William y Forsyth, Christopher, Administrative law (10 a edición, Oxford University Press, Oxford, 2009), p. 3 quienes, para nada casualmente, dedican las primeras palabras de su obra a ilustrar el proceso de 'ensanchamiento' de la Administración (Government) que caracterizó, con diversas intensidades, la casi totalidad del siglo XX del Reino Unido. 
surgimiento de estatutos normativos particulares, es decir, especiales dentro de la especialidad administrativa. Dichos estatutos vinieron a fracturar la uniformidad del "régime administratif", caracterizado, de un lado, por la actuación unilateral y autoritativa de la Administración fundada en la necesidad de salvaguardar el "intérêt général" y, del otro, en la unidad de sus órganos ${ }^{35}$.

Los servicios prestados por el Estado, aun pudiendo ser (y, en muchos casos, efectivamente siendo) de naturaleza comercial o industrial, continuaron viéndose calificados como "públicos" y, en razón de ello, siendo sometidos a una disciplina de naturaleza administrativa. Ello acentuó el proceso de "contaminación" del derecho administrativo, produciéndose una suerte de "mestizaje" entre el sujeto (público) y la actividad (comercial o industrial) que éste era llamado a desarrollar.

De esta suerte, el mayor número de manifestaciones de la actividad pública tuvo consecuencias en el plano funcional y organizativo de la Administración del Estado. La proliferación de sus actividades aumentó la variedad de las disciplinas a las que ellas se vieron sometidas; el crecimiento cuantitativo de los sujetos encargados de llevar a cabo tales nuevas actividades produjo la pérdida de la monolítica unidad de la Administración y la consecuente disgregación del sujeto unitario creado por la Revolución Francesa ${ }^{36}$.

La mayor y más intensa presencia estatal en la vida social, manifestada en la actividad de prestación de servicios y, con ellos, el aumento de la intervención pública en la vida económica, trajo como resultado que la dicotomía, la contraposición entre los universos de "lo público" y de "lo privado" - clara y definida en el derecho administrativo decimonónico se debilitara, ofuscándose, haciéndose menos clara y, sobre todo, menos definitiva ${ }^{37}$.

${ }^{35}$ Como sabemos y nos lo recuerda CASSESE, Sabino, Le amministrazioni pubbliche in Europa: per uno studio storico-comparato del diritto amministrativo en $\mathrm{EL}$ MISMO, Il diritto amministrativo: storia e prospettive (Giuffrè, Milano, 2010), pp. 331342, «il diritto amministrativo si presenta, in origine, come un diritto derogatorio, speciale, 'esorbitante' (come dicono i francesi), rispetto al 'diritto comune'. Sicché un'obbligazione pubblica è diversa da quella regolata dal codice civile, diverso è il rapporto di lavoro pubblico rispetto a quello privado, il demanio pubblico è diverso dalla proprietà privata, etc.»

${ }^{36}$ Mannori, Luca - Sordi, Bernardo, Storia del diritto amministrativo (4a edición, Laterza, Roma-Bari, 2006), p. 483.

${ }^{37}$ En el crepúsculo del siglo XIX, Otto Mayer, con su Deutsches Verwaltungsrecht, de 1895, entregó a la dogmática europea una potente lectura de síntesis del fenómeno administrativo; un modelo jurídico que era expresión de la separación, de la simplicidad y del carácter prevalecientemente autoritativo de la Administración 
Es en este momento histórico que la Administración abandona la forma de actuación principalmente autoritativa, con la cual fue caracterizada en las grandes construcciones dogmáticas del siglo XIX. La Administración del Estado se descubrió a sí misma inmersa en el mundo "material" del servicio a los ciudadanos, viéndose transformada en una "Administración prestacional". A la administración autoritativa, destinada a funcionar en la lógica dialéctica entre autoridad y libertad se sumó en modo definitivo una administración de tipo servicial ${ }^{38}$; a la administración indirecta, meramente jurídica, se unió la administración directa, capaz de llevar a la realidad, por sí misma, los fines cuya consecución le encomienda el ordenamiento jurídico ${ }^{39}$.

De este modo, con el surgimiento de la actividad prestacional, el Estado dejó de ser, para siempre, el "vacío y formal" Estado de derecho liberal ${ }^{40}$.

Este estado de cosas, sin embargo, ha cambiado sustancialmente: lenta e inexorablemente se ha alcanzado el extremo final de la evolución que se comenzó a describir en los primeros decenios del siglo XX.

En efecto, como sostiene la doctrina, los problemas financieros y de gestión del sector público causados por la "sobrecarga gubernativa" aconsejan que el Estado renuncie, en muchos ámbitos, a la administración directa ${ }^{41}$. Por tal razón, en continuidad con algunos de los postulados políticos del conservadurismo británico (que, en lo relevante para este artículo, fueron

decimonónica. El desarrollo impetuoso de la administración servicial, según Sordi, señala la completa transformación de dicho modelo dogmático. Estas ideas en Sordi, Tra Weimar e Vienna, cit. (n. 33), p. 9.

${ }^{38}$ Mameli, cit. (n. 5), pp. 347-349; ManNORI - Sordi, cit. (n. 36), pp. 491-492.

${ }^{39}$ Las categorías de "administración indirecta” ("mittelbare Verwaltung”) y "administración directa" ("unmittelbare Verwaltung") fueron identificadas, en 1923, por Kelsen en el trabajo Die Lehre von der drei Gewalten oder Funktionen des Staates (hoy en Kelsen, Hans, Die Lehre von der drei Gewalten oder Funktionen des Staates, en BRocKMÖLler, Annette (editora), Hundert Jahre Archiv für Rechts und Sozialphilosophie (1907-2007). Archiv für Rechts und Sozialphilosophie Beihefte (Franz Steiner Verlag, Stuttgart, 2007), p. 30-64. Trabajamos con la traducción italiana: KeLSEN, Hans, La dottrina dei tre poteri o funzioni dello Stato en GERACI, Carmelo (traductor y editor), Il primato del Parlamento (Milano, Giuffrè, 1982), pp. 77-120). El autor sostuvo que los fines que el ordenamiento jurídico impone alcanzar a la Administración, se puedan obtener en modo indirecto, es decir, con actos (jurídico-administrativos) dirigidos a conseguir del destinatario una determinada conducta; actos que constituyen la especificación y aplicación de normas generales, o bien, en modo directo, vale a decir, a través de la ejecución por parte de la misma administración de las conductas necesarias para el cumplimiento de los fines impuestos por el ordenamiento jurídico.

${ }^{40}$ ManNori - Sordi, cit. (n. 36), p. 481.

${ }^{41}$ CASSESE, Sabino, Le basi del diritto amministrativo (6 ${ }^{a}$ edición, Milano, Garzanti, 2000), p. 10. 
reseñados en el apartado precedente), el continente europeo se ha visto empeñado en un proceso de reducción de la esfera pública ${ }^{42}$, que no puede que verse vinculado a los procesos de integración europea, por un lado, y de globalización económica mundial, por otro. El aspecto más saliente de este proceso de redimensionamiento del ámbito de acción de "lo público" es la reformulación del concepto de interés colectivo, el cual, en algunos ámbitos, ha dejado de ser exclusivamente determinado por una decisión del poder estatal.

La identificación y, sobre todo, la satisfacción del interés colectivo hoy se alcanza reconociendo relevancia a la actuación privada, entre otras formas, a través de acuerdos con la Administración del Estado que, esta vez, actúa en un plano paritario como contraparte contractual del administrado ${ }^{43}$, al punto que se afirma que la supremacía originaria del derecho administrativo viene erosionada, ya que ella y la unilateralidad y carácter potestativo, que eran sus consecuencias, se ven sustituidas por el consenso y la bilateralidad en la actuación administrativa ${ }^{44}$.

Con el "fenómeno" de la colaboración público-privada quedan de manifiesto los nuevos papeles que, en las sociedades europeas, parecen haber asumido el sujeto público y el sector privado. Este fenómeno "sólo puede incardinarse en un contexto más amplio de cuestionamiento del paradigma tradicional de la gestión de los asuntos públicos y la aparición de un nuevo modelo en el que se impone una concepción multilateral del gobierno de los ciudadanos, en la que el Estado comparte protagonismo con otros actores también llamados a la satisfacción de intereses generales" ${ }^{45}$.

Y es así como la Comisión Europea, atenta observadora de los cambios que se verifican en el contexto europeo, analizando el fenómeno que

${ }^{42}$ Mazzamuto, Marco, La riduzione della sfera pubblica (Torino, Giappichelli, 2000).

${ }^{43}$ PICOZZA, Eugenio, Introduzione al diritto amministrativo (Padova, CEDAM, 2006), p. 14. Además, según Fioravanti, cit. (n. ), p. 76, estas transformaciones hacen que el Estado deje de presentarse como un poder que baja desde lo alto, para transformarse en un poder que se construye con la colaboración, con la negociación y con el contrato. En contra, CASSESE, Sabino, Le trasformazioni del diritto amministrativo dal XIX al XXI secolo, en Rivista Trimestrale di Diritto Pubblico (2002), p. 27-40, hoy en CASSESE, Sabino, Le trasformazioni del diritto amministrativo dal XIX al XXI secolo en EL Mismo, Il diritto amministrativo: storia e prospettive (Milano, Giuffrè, 2010), p. 487, para quien la difusión de formas de colaboración y de consenso entre la autoridad pública y los particulares no pueden significar simetría en sus posiciones jurídicas.

${ }^{44}$ CASSESE, Sabino, Le trasformazioni del diritto amministrativo dal XIX al XXI secolo, cit. (n. 43), p. 486, pero con la reserva ya anotada (p. 487).

${ }^{45}$ Dorrego de Carlos - Martínez Vásquez, cit. (n. 14), p. 32. 
nos ocupa constató que "el desarrollo de la colaboración público-privada forma parte de la evolución más general del papel del Estado en el ámbito económico, al pasar de operador directo a organizador, regulador y controlador de la actuación de sujetos privados que realizan actividades de interés público" 46 .

Por lo demás, la misma Comisión había ya elaborado una reflexión similar con ocasión de la Comunicación interpretativa sobre las concesiones en el derecho comunitario. En ésta, la Comisión se refiere al resurgimiento de las concesiones como modalidad de ejecución de grandes infraestructuras, luego que la participación del sector privado en dicha área disminuyera, a partir del primer cuarto del siglo XX, como consecuencia de la decisión de los Estados de realizar y gestionar directamente los equipamientos y los servicios públicos. La Comisión, junto a la doctrina, en otras palabras, reconoce en esta tendencia una suerte de movimiento pendular del interés estatal por aprovechar la participación del sector privado en el desarrollo infraestructural ${ }^{47}$.

A la luz de lo anterior, y retomando las palabras del Libro Verde sobre la CPP, se debe señalar que las razones que motivaron a la Unión Europea para analizar el fenómeno de la colaboración público-privada e impulsar el uso por parte de sus Estados miembros de los instrumentos jurídicos a través de los cuales ella puede canalizarse, pueden ser compendiadas en tres ideas centrales: primero, la necesidad de contención del endeudamiento del sector público; segundo, la convicción acerca de la mayor eficiencia que representa para la ejecución de los proyectos la intervención del sector privado en las diversas fases de su desarrollo y, tercero, el cambio de paradigma en la relación entre Estado y sistema económico.

\section{CONCLUSIONES}

Como se vio durante el desarrollo de este trabajo, la Unión Europea ha intentado sistematizar las razones para promover entre sus Estados miembros la participación del sector privado en la ejecución de infraestructuras a través de fórmulas contractuales que, en sentido lato, se corresponden con nuestras concesiones de obras públicas. Lo ha hecho a través de instrumen-

${ }^{46}$ Comisión de las Comunidades Europeas, "Libro Verde sobre la CPP”, cit. (n. 18) párr. 3.

${ }^{47}$ Dorrego de Carlos - Martínez Vásquez, cit. (n. 14), p. 32; Yescombe, cit. (n. 22), p. 5; UNCITRAL, Guía Legislativa sobre proyectos de infraestructura con financiación privada, Documento A/CN.9/SER.B/4 (New York, Naciones Unidas, 2000), párrs. 1-3; Estrella Faria, José Angelo, The Uncitral Legislative Guide on Privately Financed Infrastructure Projects, en The Journal of World Investment (2002), p. 211. 
tos no normativos, como el Libro Verde sobre la CPP del año 2004, que motivó una amplia reflexión cuyas conclusiones se han visto traducidas en la reciente norma comunitaria sobre los contratos de concesión (directiva 2014/23/CE). Entre uno y otro acto transcurrieron diez años, tiempo que permitió que los operadores del mercado de las obras públicas, los gobiernos y las comunidades académicas y profesionales analizaran y discutieran sobre el mejor modo de regulación de la colaboración entre el sector público y privado, específicamente, sobre aquella forma de colaboración que se ve traducida en las concesiones de obras públicas.

Nuestro país ha estado alejado de una reflexión de similar naturaleza, entidad y duración. Contamos con un sistema concesional consolidado, pero que se ha desarrollado en modo espontáneo, sin una reflexión profunda y amplia: nuestro sistema concesional es exitoso y, no obstante, parece funcionar al margen de la "legitimación" que la Presidente de la República demanda, al menos, de frente a la población.

En razón de lo anterior, si desea construirse un discurso justificativo del sistema podría recurrirse a las reflexiones desarrolladas en contextos que, si bien son distantes y distintos, presentan desafíos similares a los de nuestro país: restricciones presupuestarias y cambios en la comprensión del rol del Estado, al menos, en materia infraestructural.

Las razones que adujo la Comisión Europea para impulsar el uso de las concesiones por parte de los estados miembros de la Unión Europea, a cuyo análisis se ha dedicado este trabajo, podrían ser invocadas, mutatis mutandi, como parte de un argumento justificativo de nuestro sistema de concesiones de obras públicas.

Las importantes limitaciones presupuestarias que Chile enfrentaba a inicios de los años 90 y que hoy enfrenta Europa, a causa de las restricciones al endeudamiento público establecidas por la política monetaria de la Unión, es uno de los motivos que movió al Estado a incentivar la participación privada en el desarrollo de las primeras infraestructuras concesionadas. La muy limitada capacidad presupuestaria que sufría nuestro país durante los primeros años del programa de concesiones de obras públicas se encuentra debidamente documentada ${ }^{48}$.

Por su lado, la confianza que el Estado deposita en los empresarios del sector privado para la realización de infraestructuras fundamentales e, incluso, estratégicas para el desarrollo económico y social del país, han sido interpretadas como una manifestación del principio de subsidiaridad consagrado en nuestra Constitución. Esto se condice con el razonamiento

${ }^{48}$ Por todos, Engel, Eduardo y otros Licitación de carreteras en Chile, en Revista Estudios Públicos, 61 (1996), p. 11. 
acerca del "cambio de paradigma" del rol del Estado en la sociedad que se ha vivido también en Europa. La doctrina nacional justifica la política pública de concesiones con el rol subsidiario que el Estado habría asumido en nuestro sistema económico, principio que indicaría que el sector público debiera abstenerse de desarrollar aquellas actividades que el privado es capaz de realizar, en condiciones ventajosas para el conjunto de la comunidad ${ }^{49}$.

Sin embargo, en nuestra doctrina jurídica se echa en falta una reflexión sobre el impacto que las concesiones pueden tener en la calidad de la gestión de los servicios públicos que se prestan gracias a las infraestructuras ejecutadas, cuestión que, como hemos visto, es una de las razones centrales para la promoción de este tipo de relaciones jurídicas Estado/sector privado, al menos, en Europa. Esta reflexión parece especialmente relevante en un momento, como el presente, en el cual comienzan a ejecutarse los contratos de proyectos cada vez más complejos desde el punto de vista del impacto social de los servicios que se pretenden entregar. Se está pensando, puntualmente, en las concesiones de infraestructura hospitalaria.

En otras palabras, nuestro país se encuentra viviendo una evolución en sus concesiones que debiera ir acompañada de un análisis sobre las mejoras que se pueden o esperan lograr en la gestión de los servicios públicos. Es, de frente a este déficit, que la experiencia europea puede servir de estímulo para una reflexión sobre hacia dónde puede avanzarse en el mercado de las asociaciones público-privadas. Este trabajo no pretende dar una respuesta a tales interrogantes, sino que servir de estímulo para que la doctrina de derecho público reflexione sobre la conveniencia o inconveniencia de delegar ciertas funciones del sector público en los titulares de aquellas futuras y eventuales concesiones.

Aquello que resulta relevante destacar es que el marco conceptual de estos contratos debe ser adecuadamente analizado, con el fin de otorgar una justificación plausible para la que se presenta como una importante cesión de tareas del Estado a favor de los privados. Y es precisamente a este respecto que la demanda de un discurso de legitimación cobra plena vigencia.

En efecto, si bien es cierto que el Estado puede encomendar la rea-

${ }^{49}$ Ramírez Arrayas, José Antonio, Concesiones de obras públicas. Análisis de la institucionalidad chilena (Santiago, LegalPublishing - Abeledo Perrot, 2010), p. 5. Sobre la interpretación neoliberal del principio de subsidiaridad, LOO GUTIÉRREZ, Martín, La disciplina constitucional del principio de subsidiariedad en Italia y en Chile, en Revista de Derecho de la Pontificia Universidad Católica de Valparaíso, 23 (2009), pp. 391-426. Sobre la influencia del principio de subsidiaridad en la lectura europea de la asociación público-privada, Loo GuTiérREz, Martín, I principi di sussidiarietà e solidarietà nel partenariato pubblico-privato contrattuale (Roma, Aracne, 2014). 
lización de tareas de desarrollo y explotación infraestructural al sector privado, debe analizarse adecuadamente si tal delegación puede extenderse a funciones en las cuales la garantía de la función estatal desarrollada, precisamente, por órganos del Estado parece ser relevante (de nuevo, piénsese en las concesiones de infraestructura hospitalaria, o en las ya consolidadas concesiones de infraestructura penitenciaria, donde la "mixtura" de público y privado resulta manifiesta).

La conclusión que se quiere hacer manifiesta es que este tipo de asociaciones entre el sector público y privado son muestra de que el Estado y el sector privado pueden actuar conjuntamente en tareas de satisfacción de necesidades colectivas o públicas, toda vez que el primero dejó de ser el único sujeto llamado a la satisfacción del tales necesidades de "servicio público" y, el segundo, es impulsado a adquirir responsabilidades que lo mueven a equilibrar la búsqueda de la rentabilidad económica de las inversiones con la adecuada satisfacción del interés general.

El fenómeno jurídico que representan las asociaciones público-privadas sirve para formular una nueva lectura de los institutos más tradicionales del derecho administrativo, en primer lugar, el contrato público. Éste deja de ser concebido como una regulación de intereses negociales contrapuestos en el que, a causa del interés público involucrado, el Estado mantiene una posición predominante y absoluta sobre el contratista privado. La colaboración entre las partes de estos contratos, en el diseño, financiamiento, ejecución y explotación de las obras públicas necesarias al desarrollo social y económico, obligan a revisar las concepciones que ven en el Estado sólo una manifestación de la soberanía y del poder, transformándolo en un actor que se vale de la imprescindible cooperación y colaboración del sector privado en la consecución de las finalidades de relevancia pública que le son características, materia en la cual el sujeto público adquiere la responsabilidad de control y de garantía del correcto actuar del sector privado $^{50}$, a través de la identificación de los fines que deben ser satisfechos y del adecuado empleo de los medios con los cuales ellos se satisfacen.

Creemos que sólo una adecuada inteligencia de las razones de este fenómeno hará plausible la (demandada) legitimación del sistema.

\section{BibLIOGRAFÍA}

Akitoby, Bernardin - Hemming, Richard - Schwartz, Gerd, Public Investment and Public-Private Partnerships, en International Monetary Fund Economic Issues, 40 (International Monetary Fund, New York, 2007).

${ }^{50}$ MANNORI - Sordi, cit. (n. 36), p. 522. 
Allen, Grahame, The Private Finance Initiative, House of Commons Library Research Paper 3/79 (House of Commons, London, 2003).

Bobbio, Norberto, La grande dicotomia: pubblico/privato, en EL MISMO, Stato, governo, società ( $2^{a}$ edición, Einaudi, Torino, 1995).

Bovis, Christopher, EU Public Procurement Law (Cheltenham, UK - Northampton, USA, Edward Elgar Publishing, 2007).

Bovis, Christopher, The Development of Public Private Partnerships at European Level, en European Public Private Partnership Law Review (2006).

CASSESE, Sabino, La costituzione economica europea en Rivista Italiana di Diritto Pubblico Comunitario (2001).

CASSESE, Sabino (editor), La nuova costituzione economica (4a edición, Laterza, Roma - Bari, 2007).

CASSESE, Sabino, La "vecchia" costituzione economica: i rapporti tra Stato ed economia dall Unità ad oggi, en EL MISMO (editor), La nuova costituzione economica (4a edición, Laterza, Roma - Bari, 2007).

CASSESE, Sabino, Le amministrazioni pubbliche in Europa: per uno studio storicocomparato del diritto amministrativo, en EL MISMO, Il diritto amministrativo: storia e prospettive (Milano, Giuffrè, 2010).

CASSESE, Sabino, Le basi del diritto amministrativo (6a edición, Milano, Garzanti, 2000).

CASSESE, Sabino, Le trasformazioni del diritto amministrativo dal XIX al XXI secolo, en EL MISMO, Il diritto amministrativo: storia e prospettive (Milano, Giuffrè, 2010).

Chiт, Mario Pilade, I partenariati pubblico-privati e la fine del dualismo tra diritto pubblico e diritto comune, en EL MISMO (editor), Il partenariato pubblico-privato. Concessioni, finanza di progetto, società miste, fondazioni (Napoli, Editoriale Scientifica, 2009).

Cori, Rosalba - Giorgiantonio, Cristina - Paradisi, Ilaria, Allocazione dei rischi e incentivi per il contraente privato: un" analisi delle convenzioni di project financing in Italia en AA.VV., Le infrastrutture in Italia: dotazione, programmazione, realizzazione (Roma, Banca d'Italia, 2011).

Del SAZ, Silvia, La nueva ley de contratos del sector público. ¿Un nuevo traje con las mismas rayas? en Revista de Administración Pública, 174 (2007).

Dorrego de Carlos, Alberto - Martínez Vásquez, Francisco, La colaboración público-privada en Los MISMOS (editores), La colaboración público-privada en la Ley de contratos del sector público (Madrid, La Ley, 2009).

ENGEL, Eduardo - FisCHER, Ronald - GALETOVIC, Alexander, Licitación de carreteras en Chile, en Revista Estudios Públicos, 61 (1996).

Engel, Eduardo - FisCher, Ronald - GAletovic, Alexander, The Economics of PublicPrivate Partnerships. A Basic Guide (New York, Cambridge University Press, 2014).

Estrella Faria, José Angelo, The Uncitral Legislative Guide on Privately Financed Infrastructure Projects, en The Journal of World Investment (2002).

FioraVAnti, Maurizio, Per una storia dello Stato moderno in Europa, en Quaderni Fiorentini per la Storia del Pensiero Giuridico Moderno, 39 (2010).

Freeman, Jody - Minow, Martha (editores), Government by Contract: Outsourcing and American Democracy (Cambridge, Mass. - London, Harvard University Press, 2009).

Frilet, Marc - Lager, Florent, Public Procurement Issues in the European Union, en European Business Law Review (2010). 
García Capdepón, Pedro María, Los contratos de colaboración público-privada, en Revista Española de la Función Consultiva, 3 (2005).

Giorgiantonio, Cristina - Giovanniello, Valentina, Infrastrutture e "project financing" in Italia: il ruolo (possibile) della regolamentazione, en AA.VV., Le infrastrutture in Italia: dotazione, programmazione, realizzazione (Roma, Banca d'Italia, 2011).

González García, Julio, Contrato de colaboración público-privada, en Revista de Administración Pública, 170 (2006).

Grimshaw, Adam - Vincent, Steve - Willmont, Hugh, Going Privately: Partnership and Outsourcing in UK Public Services, en Public Administration, 80 (2002).

Hoppea, Eva - Schmitz, Patrick, Public-Private Partnerships versus Traditional Procurement: Innovation Incentives and Information Gathering, en SSRN eLibrary (2012).

International Monetary Fund (Fiscal Affairs Department), Public-Private Partnerships (Washington (DC., 2004).

KeLSEn, Hans, Die Lehre von der drei Gewalten oder Funktionen des Staates, en BrocKMÖLLER, Annette (editora), Hundert Jahre Archiv für Rechts und Sozialphilosophie (1907-2007). Archiv für Rechts und Sozialphilosophie Beihefte (Stuttgart, Steiner, 2007).

Kelsen, Hans, La dottrina dei tre poteri o funzioni dello Stato, en Geraci, Carmelo (traductor y editor), Il primato del Parlamento (Milano, Giuffrè, 1982).

LOO GuTIÉRREZ, Martín, I principi di sussidiarietà e solidarietà nel partenariato pubblicoprivato contrattuale (Roma, Aracne, 2014).

Loo GutiérRez, Martín, La disciplina constitucional del principio de subsidiariedad en Italia y en Chile, en Revista de Derecho de la Pontificia Universidad Católica de Valparaiso, 23 (2009).

Mamel, Barbara, Servizio pubblico e concessione. L'influenza del mercato unico sui regimi protezionisti e regolamentati (Milano, Giuffrè, 1998).

Mannori, Luca - Sordi, Bernardo, Storia del diritto amministrativo (4a edición, Roma - Bari, Laterza, 2006).

Mazzamuto, Marco, La riduzione della sfera pubblica (Torino, Giappichelli, 2000).

Napolitano, Giulio, Pubblico e privato nel diritto amministrativo (Milano, Giuffrè, 2003).

Nieto Garrido, Eva, El "New Public Management" y el Gobierno a través de contratos en el Reino Unido, en Revista de Administración Pública, 162 (2003).

NiEto Garrido, Eva, La financiación privada de obras y servicios públicos en el Reino Unido (de Private Finance Initiative a Public Private Partnerships), en Revista de Administración Pública, 164 (2004).

Public-Private Partnerships: In Pursuit of Risk Sharing and Value for Money (Paris, OECD Publishing, 2008).

Transport Infrastructure Investment. Options for Efficiency (Paris, OECD Publishing, 2008).

PICOZZA, Eugenio, Introduzione al diritto amministrativo (Padova, CEDAM, 2006).

Ramírez Arrayas, José Antonio, Concesiones de obras públicas. Análisis de la institucionalidad chilena (Santiago, LegalPublishing - Abeledo Perrot, 2010).

SADKA, Efraim, Public-Private Partnerships: A Public Economics Perspective, en SSRN eLibrary (2006).

SORDI, Bernardo, Servizi pubblici e concorrenza: su alcune fibrillazioni tra diritto comunitario e tradizione continentale, en Quaderni Fiorentini per la Storia del Pensiero Giuridico Moderno, 31 (2002). 
SORDI, Bernardo, Tra Weimar e Vienna. Amministrazione pubblica e teoria giuridica nel primo dopoguerra (Milano, Giuffrè, 1987).

UNCITRAL, Guía Legislativa sobre proyectos de infraestructura con financiación privada, Documento A/CN.9/SER.B/4 (New York, Naciones Unidas, 2000).

WADE, William - ForsYTH, Christopher, Administrative Law (10ª edición, Oxford, Oxford University Press, 2009).

Yescombe, Edward. R., Public-Private Partnerships: Principles of Policy and Finance (Oxford, Butterworth Heinemann, 2007). 
